\title{
Digital Asset Management
}

\author{
Fulvio Re Cecconi, Mario Claudio Dejaco, Nicola Moretti, \\ Antonino Mannino and Juan Diego Blanco Cadena
}

\begin{abstract}
Digital Asset Management is a key discipline enabling a sustainable and high-quality built environment. The physical asset is nowadays more and more integrated within the digital environment, therefore it produces a great amount of information during its life cycle. This information should be used to improve process management during the use phase of the asset, according to a servitised and crossdisciplinary approach. Accordingly, a methodological framework for asset management business processes reengineering is here presented. Through the application of the proposed set methods and procedures, it is possible to leverage innovative Information and Communication technologies (ICTs) for the development of improved information management practices in digital built environment management. The case studies developed demonstrated the possibility to effectively implement innovative Digital Asset Management processes and address different core areas of the discipline.
\end{abstract}

\section{Shifting Towards a New Real Estate Market}

The real estate market is shifting its scope form the traditional meaning of the asset, conceived as tangible good exploited as a means for achieving a primary objective, to the integration of physical assets into the set of services delivered to the client (Moretti et al. 2017). This new way of thinking about the real estate, brings tangible and intangible goods and services together, as a whole new product to be sold on the market. The output of the real estate development industry becomes a system of both project components and added services (Baines et al. 2008). Moreover, management of the built environment has been characterised over the last years by an increase in the complexity of physical assets, as well as the high number of stakeholders and

F. Re Cecconi $(\varangle) \cdot$ M. C. Dejaco · N. Moretti · A. Mannino - J. D. Blanco Cadena Architecture, Built Environment and Construction Engineering-ABC Department, Politecnico di Milano, Milan, Italy

e-mail: fulvio.rececconi@polimi.it 
pervasive use of Information Communication Technologies (ICTs) (Centre for Digital Built Britain 2018). Physical assets can be considered complex systems featuring tangible and intangible performances. The shift from the traditional paradigm (the building as a product) to the contemporary one (the building as a service) is enabled by the digitisation leading to a new complexity, to be managed through approaches enabling modelling and management of information, for achieving a more sustainable built environment. These dynamics raise the issue of how digital-based processes can be encompassed within AM and which are the most suitable tools and practices to be employed to catch the new complexity of the built environment. Therefore, the research triggers process innovation for Asset Management, exploiting existing tools and practices, combined and reshaped to achieve enhanced performances of the built environment.

\section{State of the Art}

The built environment is currently more and more integrated with the digital environment, enabling new processes in the asset management. The Internet of Things implementation in Architecture, Engineering, Construction and Operation (AECO) is gaining momentum, allowing the sector to reach advanced building performances (Wong et al. 2018). Higher level of effectiveness and automation for comfort control and adjustment (Fan and Xia 2015), continuous commissioning and maintenance management of physical assets (Dixit et al.2019) are only a couple of the features that can be achieved through the integration of the physical asset with a dense network of sensors and actuators and with automated systems. The building, in this context, must be considered as an entity, able to recognise external and internal changes and adapt its behaviour to provide users with defined performance levels. These information exchanges between the user and the building are typical of the cognitive building, namely an asset which is able to adapt itself according to the behaviour of the users who, conversely, receive information from the asset and can be involved in the control loop in a bi-directional way (Rinaldi et al. 2016). The great number of sensors, actuators and advanced automated systems that characterise the contemporary built environment raises new issues concerning the management of the wide information flow (Boton et al. 2015). Accordingly, IT tools should be exploited to tackle cross-sector problems, since traditional approaches are no longer suitable with the increasing complexity of the built environment. Therefore, methodologies for analysis and interpretation of Big Data could be exploited for handling the unstructured, real-time data flows, to be interpreted and processed in order to achieve a higher knowledge and effectiveness in asset management.

Structured information can be integrated in the Building Information Modelling approach which, according to ISO 19650-2:2018 (2018), can be intended as a digital object-oriented process for design, construction and management of buildings or infrastructures. BIM enables professionals to make informed decisions thanks to reliable and updated information, during the entire life cycle of the asset (Sanchez 
et al. 2016). Accordingly, BIM cannot only be considered as a 3D-oriented design methodology, but as a digitally based methodology, exploiting different Information and Communication Technology (ICT) tools and techniques, which allow to effectively manage the physical assets in a sustainable way.

\section{Aim of the Research}

The ongoing research at the Politecnico di Milano-ABC Dept. aims at enhancing Asset Management business processes, through new information management approaches enabled by availability of ICT tools. The employment of this innovative approach allows for the optimisation of existing Asset Management processes and the creation of new ones. Therefore, a methodological framework to improve or develop new Digital Asset Management processes has been developed. The methodological framework is articulated in three phases, bringing from a strong knowledge and understanding of the traditional Asset Management processes, to the optimisation or development of new Digital Asset Management ones.

\section{Methods and Tools}

The methodological approach is organised in three steps: the AM process mapping, the process modelling and the process reengineering. The first concerns the identification and categorisation of the AM core functions, the second implies a standardised methodology for business process modelling, the third concerns the optimisation and creation of new digital-based and servitised AM processes.

AM encompasses a wide array of processes and sub-processes to be implemented in order to achieve acceptable levels of performance and maintain their value during their entire life cycle. Engineering Asset Management can be divided into 14 areas, classified by typology (strategic, tactical, operational) and according to the lifecycle phases in which they take place. Processes in Fig. 1 are the most frequently implemented in asset management. The business process mapping is the first step to achieve a strong knowledge of the AM discipline and to define the boundaries of Digital Asset Management: it allows us to identify processes to be managed in order to make informed decisions on assets.

Each area is composed by a sequence of input, core processes, sub-processes and output. The Business Process Modelling (BPM) technique has been adopted to model the AM business processes, identifying the information flows among processes. Therefore, for each process identified in the business process mapping phase, inputs, main processes, sub-processes and outputs are modelled, to classify the main flows (relationships) and transformations (activities). Modelling of the information flows of core AM functions enables the digital-based servitised reengineering which takes places in the third phase. 


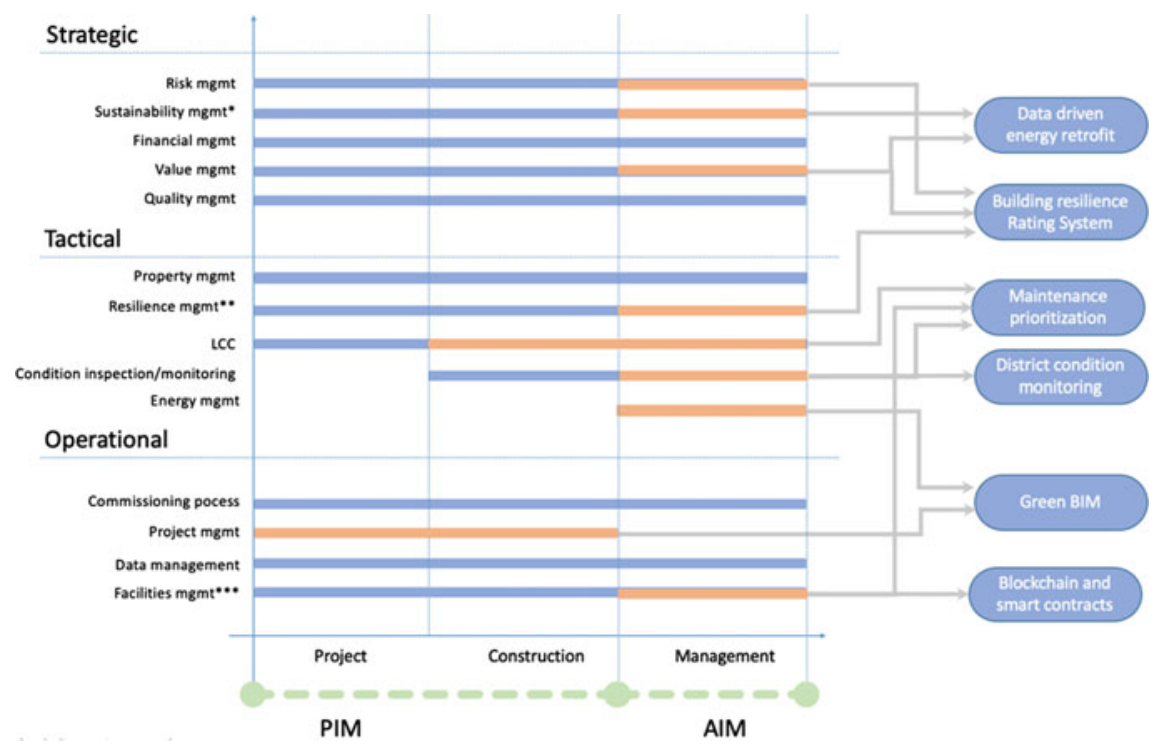

Fig. 1 Digital Asset Management core areas and reengineering outcomes (case study)

The third phase concerns the Business Process Reengineering. Reengineering means the optimisation or creation of new business processes, in order to achieve a digitally based and servitised outcome.

This operation may lead to the complete redevelopment of the processes previously employed by the organisation. In this phase, the identification of a number of additional key features of the processes is crucial. The key features can be summarised as: the typology of the organisation; the operating context; the organisation's constraints (financial and regulatory); the needs of the organisation and the involved stakeholders. These five features allow to properly shape the process reengineering operations and outcomes, without losing the standardisation. To the five features a sixth must be added: the organisation's data availability and accessibility. This is a primary driver for the business process reengineering in Digital Asset Management and for achieving the benefits deriving from it (e.g. more efficiency and effectiveness, reduction in uncertainty, increased reliability, etc.). This assessment also informs on which are the most suitable processes to be reengineered. Once the definition of the organisation's context and constraints has been defined, processes can be optimised through the use of enabling ICTs. The reengineering process takes places according to the principles of modularity, scalability and cross-domain. Currently, nine core AM functions have been reengineered. 


\section{Case Studies and Tools}

Processes reengineered so far are represented in Fig. 1. Orange bars represent the reengineered processes. Some processes can be extended from a single asset to the asset portfolio, or from the asset to the neighbourhood. Therefore, the scalability principle has been employed. Moreover, different core areas have been connected to meet an inter-disciplinary and cross-domain approach.

\subsection{Artificial Neural Networks and GIS for Energy Retrofit Policy}

School buildings in Italy are outdated, in critical maintenance conditions and they often perform below acceptable service levels and quality standards. Nevertheless, data supporting renovation policies is missing or is very expensive to be obtained (Fig. 2).

This case study proposes a method for evaluating buildings' energy savings potential, using the Building Energy Certification (Certificazione Energetica degli Edifici-CENED) open database (2018). The aim of the study concerns the development of a data-driven set of methods, based on the use of open data, machine learning (ML) and Geographic Information Systems (GIS) to support regional energy retrofit policies for school buildings. The main advantage concerns the possibility to predict the post-retrofit energy savings, avoiding the expensive on-site Condition Assessment (CA) phase. Data has been first clustered to identify the most common thermo-physical properties of the envelope, then three retrofit scenarios have been defined, to allow for the retrofit of homogeneous types of buildings. The energy saving potentials have been evaluated through the implementation of eight Artificial
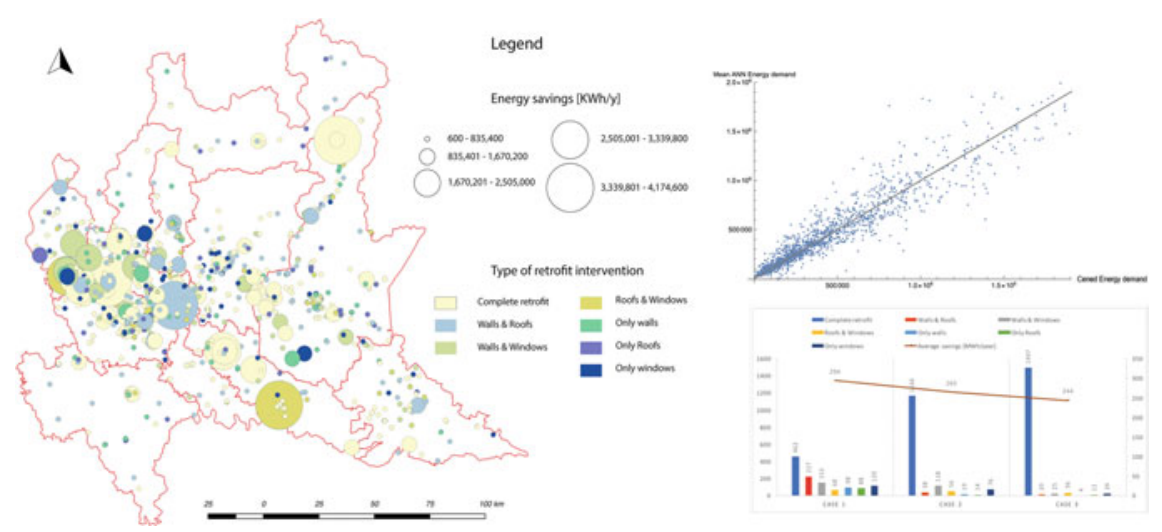

Fig. 2 School portfolio refurbishment map and scenario analysis 


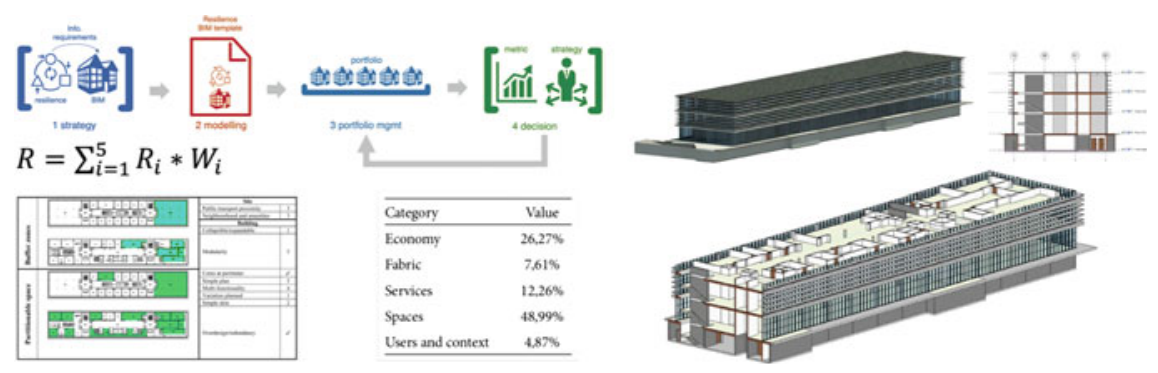

Fig. 3 Research diagram, resilience rating equation, and 3D model of the case study building

Neural Networks (ANN). Finally, data has been geolocated and further processed to support the definition of the energy retrofit policies for the most critical regional areas. The Lombardy region has been chosen as case study to test the robustness of the proposed methods. The results of the case study proved that school buildings energy retrofit policies can be supported and defined using available open data, ML and GIS. The future developments of the research concern the further integration of GIS for retrofit cost assessment and scenario analysis (Re Cecconi et al. 2019a).

\subsection{BIM-Based Building Resilience Rating System}

Measuring and rating resilience of assets is a key enabler for asset and portfolio management. Through this case study a resilience rating system for buildings has been developed by utilising a Building Information Modelling (BIM) approach. The assessment is carried out through a calculation following the Analytical Hierarchy Process (AHP). This methodology can be applied to different types of buildings, without a loss of precision or reliability. This resilience rating forms an integral part of a more comprehensive array of Key Performance Indicator (KPI) frameworks for asset and portfolio management, and therefore can significantly influence strategic investment choices for designers, engineers, and building owners (Re Cecconi et al. 2018) (Fig. 3).

\subsection{Building Maintenance Budget Allocation}

Available data on asset condition and performances can be conveyed into different Key Performance Indicators (KPIs). Many KPIs measuring technical, functional and economic/financial asset performances can be found in the literature. Nevertheless, they are often strictly related to a specific scope, thus they provide an incomplete depiction of the whole assets' performances. The objective of this case study is to provide facility managers and asset owners with an easy instrument to prioritise 

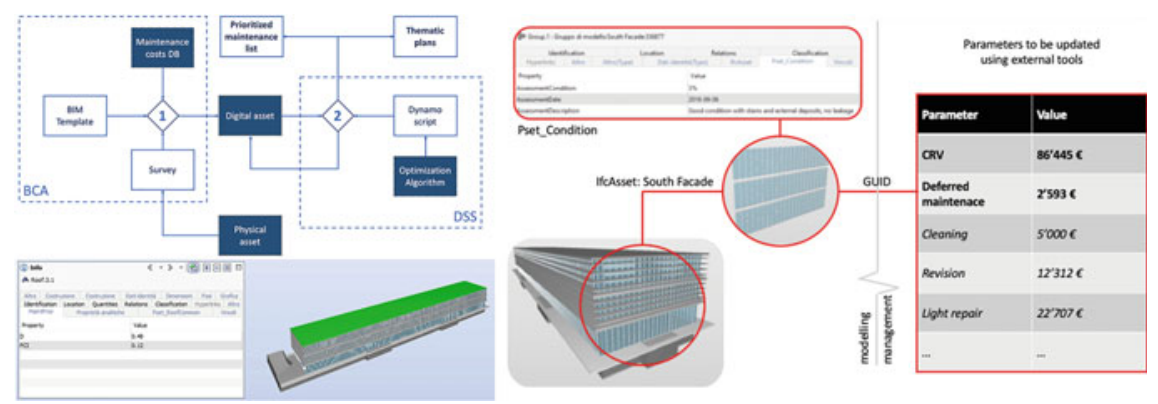

Fig. 4 Maintenance budget allocation research schema and interface

maintenance. In order to reduce costs related to its use, the instrument, developed in the form of a Decision Support System (DSS), is based on existing and reliable performance metrics and leverages new technologies like Building Information Modelling (BIM). Accordingly, the Facility Condition Index (FCI) is combined with the $\mathrm{D}$ index, a KPI related to the age of building components, developed by the authors. The joint use of the FCI and the D index, allows facility managers to make more conscious decisions. The proposed DSS helps in the definition of the best maintenance plan, providing a ranking of building components which require more urgent maintenance interventions. Although the DSS should be tested measuring its ability to preserve buildings and their performances in the long term, the first results are positive, as confirmed by the application to a case study on an office building in Italy. Moreover, the usability of the instrument has been appreciated by the users in a medium size Italian company (Re Cecconi et al. 2019b) (Fig. 4).

\subsection{Green BIM}

Rating systems are assumed as instruments to endorse architectural quality, reliability, energy efficiency, economic convenience and, finally, assign a sustainability label. Moreover, these tools can be tied to a BIM model. The aim of this case study is test a lean methodology to fulfil Common European Sustainable Built Environment Assessment (CESBA) requirements through Construction to Operations Building information exchange (COBie) in projects on existing buildings adopting the BIM Bronze approach from the UK Ministry of Justice (MoJ). This will allow to develop a semantic model and to extract sustainability reports in a post-construction phase, thereby minimising the cost of gathering information on existing buildings. An illustrated example regarding energy criteria of CESBA protocol has been developed to further the proposed approach (Maltese et al. 2017) (Fig. 5). 

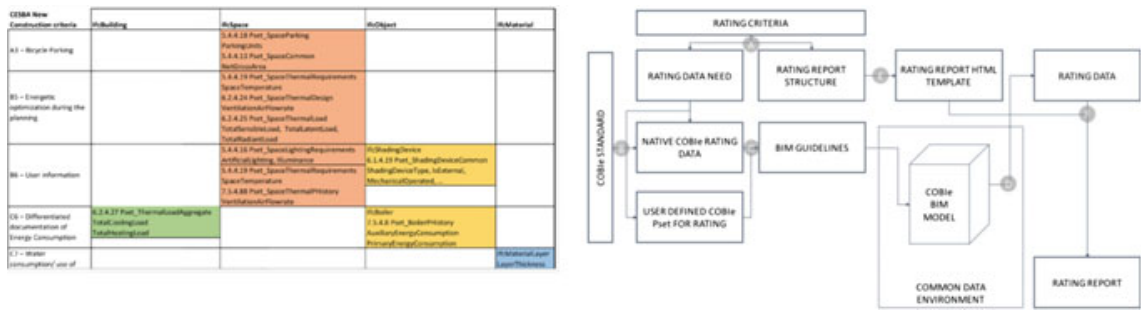

Fig. 5 Green BIM research schema and COBie mapping template

\section{Smart Contract for Optimised Facility Management of Cognitive Buildings}

Building operations require fine Facility Management (FM) when it comes to achieving satisfactory performance levels of spaces within buildings. It is known that occupancy monitoring fosters energy efficiency in buildings through dynamic adaptation of indoor conditions according to the variation in occupancy. A similar approach can enhance maintenance contract management too, especially if coupled with a contract management system such as blockchain. Here a methodology is presented for the optimisation of maintenance contracts, through occupancy monitoring with an arranged sensor network. For this, two ultrasonic sensor modules have been employed for tracking the use intensity of a corridor within an office building in Milan. Tests were performed for determining the proper configuration for their installation, to accurately read the use intensity flow. Data can be processed and stored within a digital asset model, associated with the maintenance plan. Once cleaning requirements reaches a predefined threshold, which is agreed and defined in the maintenance plan using as an indicator the occupants flow, the system triggers a maintenance alert to the contractor, who can then activate the cleaning intervention (Fig. 6).

The cleaning need threshold stored in the BIM model can be considered as the oracle of the maintenance smart contract which is activated once the cleaning operation has started. The maintenance smart contract allows users to automatically validate the transaction, once the maintenance intervention is performed and verified. Users' privacy issues are surpassed since the sensors measure the distance from the nearest object for determining the flow of people, without registering any personal information.

The proposed approach enables an enhancement for the automation of maintenance management operations in a cost-effective manner. However, further validation and trials are required with respect to the flexibility of its application (different space types), especially when dealing with abrupt occupants' displacement (Blanco Cadena et al. 2019). 


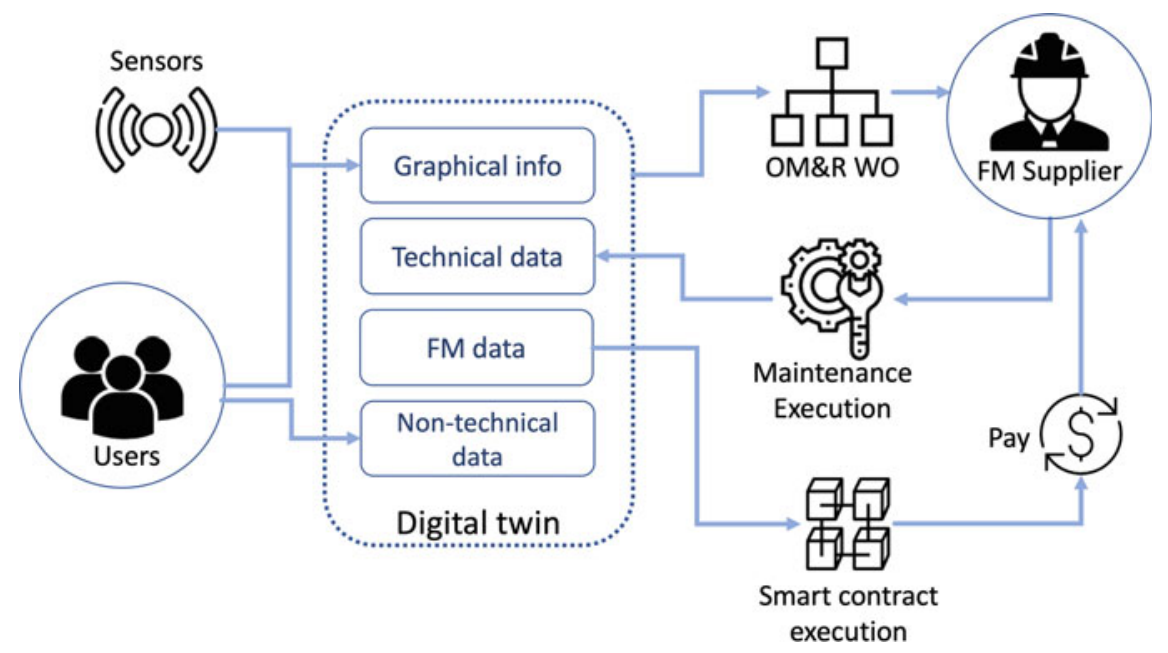

Fig. 6 Smart contracts for improved facility management research diagram

\section{Conclusions and Next Development}

The methodological approach has been defined and organised in three steps which start from the deep knowledge of AM processes leading to their reengineering, through the information flow modelling enabled by ICT technologies and tools. The digitisation potential of the processes is defined through the analysis of availability of information and takes places thanks to the modularity, scalability and cross-domain principles. This can be considered a streamlined assessment for the initialisation of a reengineering process. The proposed three-tiered methodology is the framework within which the reengineering of business processes takes place.

Through the proposed case studies, it has been demonstrated how Digital built Asset Management processes can be developed according to a data-driven and servitised approach. The case studies, which can be considered as models with an immediate business application (ready-to-use tools), demonstrate the possibility of achieving positive results in the implementation of the DAM processes. In conclusion, further applications and validation of the proposed methods could strengthen the research and business outcomes and foster new partnerships with the industry.

Acknowledgements The authors would like to acknowledge Rigamonti Francesco e Figli S.p.A. and Tekser S.r.l. for funding these studies. Moreover, the authors thank Dr. Sebastiano Maltese for supporting and contributing to this study. 


\section{References}

Baines TS, Lightfoot HW, Benedettini O, Kay JM (2008) The servitization of manufacturing: a review of literature and reflection on future challenges. J Manuf Technol Manag 20(5):547-567

Blanco Cadena JD, Moretti N, Poli T, Re Cecconi F (2019) Low-cost sensor network in cognitive buildings for maintenance optimisation. Tema: Technol Eng Mater Archit 5(1):93-102. ISSN 2421-4574, https://doi.org/10.17410/tema.v5i1.218

Boton C, Halin G, Kubicki S, Forgues D (2015) Challenges of big data in the age of building information modeling: a high-level conceptual pipeline. Lecture Notes in Computer Science (including subseries Lecture Notes in Artificial Intelligence and Lecture Notes in Bioinformatics), vol 9320, pp 48-56

Centre for Digital Built Britain (2018) Year one report. Towards a digital built Britain

Dixit MK, Venkatraj V, Ostadalimakhmalbaf M, Pariafsai F, Lavy S (2019) Integration of facility management and building information modeling (BIM). Facilities [Internet]. 37(7/8):455-483. https://www.emeraldinsight.com/doi/10.1108/F-03-2018-0043

Fan Y, Xia X (2015) A multi-objective optimization model for building envelope retrofit planning. In: Yan J, Shamim T, Chou SK, Li H (eds) Energy Procedia [Internet]. Elsevier Ltd., Amsterdam, pp 1299-1304. https://www.scopus.com/inward/record.uri?eid=2-s2.0-84947063926\&doi=10. 1016\%2Fj.egypro.2015.07.193\&partnerID=40\&md5=3abdbf62a628cbe604e3f42eb80317dd

ISO. BS EN ISO 19650-2:2018 (2018) Organization and digitization of information about buildings and civil engineering works, including building information modelling (BIM) - Information management using building information modelling. Part 2: delivery phase of the assets. https://www.iso.org/committee/49180.html?fbclid=IwAR14dRLyXIH_ C3jrlfzD0Uke_HimIXeR_w5_CJoFwC2gsJAL4hrOfRLFJ20

Maltese S, Moretti N, Re Cecconi F, Ciribini ALC, Kamara JM (2017) A lean approach to enable sustainability in the built environment through BIM. Techne-J Technol Archit Environ 13:278286

Moretti N, Dejaco MC, Maltese S, Re Cecconi F (2017) The maintenance paradox. ISTeA 2017_ Re-shaping. Constr Ind 234-242

Re Cecconi F, Moretti N, Maltese S, Dejaco MC, Kamara JM, Heidrich O (2018) A rating system for building resiliencel Un rating system per la resilienza degli edifici. Techne 15:358-365

Re Cecconi F, Moretti N, Tagliabue LC (2019a) Application of artificial neutral network and geographic information system to evaluate retrofit potential in public school buildings. Renew Sustain Energy Rev [Internet] 110:266-277. https://www.sciencedirect.com/science/article/pii/ S1364032119302941?dgcid=author. Accessed 12 May 2019

Re Cecconi F, Moretti N, Maltese S, Tagliabue LC (2019b) A BIM-based decision support system for building maintenance. In: Advances in informatics and computing in civil and construction engineering [Internet]. Springer, Cham, pp 371-378. http://link.springer.com/10.1007/9783-030-00220-6_44. Accessed 5 Nov 2018

Regione Lombardia (2018) Database CENED + 2-Certificazione ENergetica degli EDificilOpen Data Regione Lombardia [Internet]. https://www.dati.lombardia.it/Energia/Database-CENED-2Certificazione-ENergetica-degli-E/bbky-sde5. Accessed 15 Nov 2018

Rinaldi S, Bittenbinder F, Liu C, Bellagente P, Tagliabue LC, Ciribini ALC (2016) Bi-directional interactions between users and cognitive buildings by means of smartphone app. In: 2016 IEEE international smart cities conference (ISC2) [Internet]. IEEE, pp 1-6. http://ieeexplore.ieee.org/ document/7580819/. Accessed 28 Feb 2018

Sanchez AX, Hampson KD, Vaux S (2016) Delivering value with BIM: a whole-of-life approach [Internet]. Delivering value with BIM: a whole-of-life approach. Taylor and Francis Inc., London, pp 1-344. https://www.scopus.com/inward/record.uri?eid=2-s2.0-84979718392\&doi=10. 4324\%2F9781315652474\&partnerID=40\&md5=d49efd9f72d1714050550ef876bbf331 
Wong JKW, Ge J, He SX (2018) Digitisation in facilities management: a literature review and future research directions. Autom Constr [Internet] 92:312-326. https://www.sciencedirect.com/ science/article/pii/S0926580517309020. Accessed 2 Nov 2018

Open Access This chapter is licensed under the terms of the Creative Commons Attribution 4.0 International License (http://creativecommons.org/licenses/by/4.0/), which permits use, sharing, adaptation, distribution and reproduction in any medium or format, as long as you give appropriate credit to the original author(s) and the source, provide a link to the Creative Commons license and indicate if changes were made.

The images or other third party material in this chapter are included in the chapter's Creative Commons license, unless indicated otherwise in a credit line to the material. If material is not included in the chapter's Creative Commons license and your intended use is not permitted by statutory regulation or exceeds the permitted use, you will need to obtain permission directly from the copyright holder.

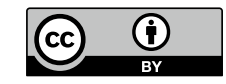

